\title{
Influencia de la concentración de nutrientes y la herviboría sobre la estructura y la función de una comunidad algal
}

\author{
María A. Rodrigo*, Carmen Rojo, José Larrosa, Matilde Segura y Marie Moeys
}

Grupo de Ecología Integrativa. Institut Cavanilles de Biodiversitat i Biologia Evolutiva. Universitat de València. Apartado Oficial 2085. 46071-València.

* Autor responsable de la correspondencia: maria.a.rodrigo@uv.es

\begin{abstract}
RESUMEN
Influencia de la concentración de nutrientes y la herviboría sobre la estructura y la función de una comunidad algal

Un mismo conjunto de algas puede generar diferentes ensamblados finales estables en función del ambiente (lago, laguna, etc.) en que se desarrollen. En este trabajo se pretende averiguar qué mecanismos están afectando simultáneamente al conjunto de especies para que se produzca una selección de ellas apropiada a cada ambiente acuático, y cómo la variabilidad de la respuesta se ve reflejada en la estructura y función de la comunidad (biomasa total, fotosíntesis y respiración). Se ha ensayado la formación de una comunidad algal a partir de la disposición simultánea de 8 especies de algas cosmopolitas de agua dulce de morfología bien distinta (Cosmarium contractum, Cryptomonas ovata, Euglena gracilis, Limnothrix redekei, Monoraphidium contortum, Pediastrum tetras, Planktothrix agardhii y Scenedesmus acutus) en distintos tipos de medio de cultivo. Los tratamientos han sido cuatro: mayor y menor concentración de nutrientes (N y P) y presencia o no de Daphnia magna, Keratella cochlearis y Brachionus calicyflorus. Se han ensayado, además, sistemas de competencia entre algas y se ha averiguado la viabilidad de estas especies como inóculos tras un período de senescencia. La presencia de herbívoros (depredación) y la concentración de nutrientes (competencia), son los mecanismos que aislada o sinérgicamente afectan al ensamblado de microalgas determinando diferentes estados alternativos desde un mismo conjunto de microalgas. Pero el efecto de estos mecanismos es a su vez diferente en función de las propiedades de las microalgas implicadas como son su tamaño, su tasa de crecimiento y la viabilidad de cada especie como inóculo. La presencia de herbívoros y la oligotrofia reducen la biomasa de productores primarios pero aumentan la diversidad relajando la competencia y aumentando la incertidumbre sobre el final alcanzado. Se demuestra además que las variaciones en la estructura de la comunidad se reflejan directamente en su función, afectando en última instancia a la producción primaria y la respiración.
\end{abstract}

Palabras clave: Reglas de ensamblado, estrategia de crecimiento, dominancia, morfología, nitrógeno, fósforo, electrodo de Clark, fotosíntesis, respiración.

\begin{abstract}
Influence of the nutrient concentration and herbivory on the structure and function of an algae community

The same pool of algae can generate different final stable assemblages depending on the environment (lake, lagoon, etc.) where they develop. In this work we attempt to ascertain the mechanisms that simultaneously affect the species to appropriately select them for each aquatic environment, and how the variability in the response is reflected in the community structure and function (total biomass, photosynthesis and respiration). An algal community has been formed assembling 8 species of freshwater cosmopolite algae which have quite different morphologies (Cosmarium contractum, Cryptomonas ovata, Euglena gracilis, Limnothrix redekei, Monoraphidium contortum, Pediastrum tetras, Planktothrix agardhii and Scenedesmus acutus) using several kinds of culture media. The treatments were four: low and high nutrient concentrations $(N$ and $P)$ and presence and absence of Daphnia magna, Keratella cochlearis, and Brachionus calicyflorus. The competition between pairs of algae has been studied and also the viability of the species as inoculum after a senescence period. The presence of herbivores (predation) and the nutrient concentration (competition) are the mechanisms that by themselves or sinergically affect the microalgae assemblages originating different alternative states from the same pool of species. But the effect of these mechanisms is different depending on the algal properties such as size, growth rate, and viability as inoculum. The presence of herbivores and the oligotrophic conditions reduce the primary producers' biomass but increase diversity due to relaxation of competition and increase the uncertainty of final states. It is also shown that the variations in the community structure are directly reflected on the function, affecting primary production and respiration.
\end{abstract}


Keywords: Assembly rules, growth strategy, dominance, morphology, nitrogen, phosphorus, Clark electrode, photosynthesis, respiration.

\section{INTRODUCCIÓN}

Dentro de las cuestiones básicas en la ecología de las microalgas están el comprender porqué se dan unas asociaciones algales y no otras en los diferentes ambientes como lagos, lagunas, etc. (Reynolds, 1998) y averiguar los factores determinantes en su estabilidad (Naselli-Flores $\mathrm{et}$ al., 2003). A partir del desarrollo de la ecología de las comunidades planctónicas ha quedado claro que la composición de una comunidad observada en una masa acuática puede estar controlada por la depredación (los procesos "desde arriba", Carpenter \& Kitchell, 1993; Ortega-Mayagoitia et al., 2002), por la competencia por los recursos (control "desde abajo", Cottingham, 1999), o por la actuación común de ambos factores (OrtegaMayagoitia et al., 2003, Cottingham et al., 2004). Además, otros factores a tener en cuenta y que explicarían la varianza observada son: la historia de formación de la comunidad (Padisák, 1992; Drake, 1991; Drake et al., 1999; Rojo et al., 2006) y la frecuencia e intensidad de las perturbaciones (Reynolds et al., 1993). La complejidad de este panorama y la relativa falta de experimentación con sistemas multiespecíficos de plancton hacen que las hipótesis surgidas de las observaciones repetidas en el campo no acaben de ser determinantes (Rojo \& Álvarez-Cobelas, 2000).

Un patrón empírico sobre la presencia de algún grupo de algas y no otros en determinados ambientes es el descrito como sucesión o sucesión estacional fitoplanctónica por Margalef (1958), Reynolds (1984), por los ecólogos del plancton (Harris, 1986; Sommer et al., 1986), para humedales (Chow-Fraser, 1998; Rojo et al., 2000) y de un modo más general, basándose en una clasificación funcional, el desarrollado en los últimos años (Olrik, 1994; Reynolds, 1997; Reynolds et al., 2002). Este patrón se basa en numerosas observaciones de campo $\mathrm{y}$ se explica por las diferentes estrategias de crecimiento de las algas, sus diferentes morfologías (tamaño, forma, etc.), movilidad y su metabolismo (fotosíntesis y utilización de recursos). De tal modo que ante un conjunto de especies de algas los investigadores especialistas deberían poder predecir qué selección de ellas sería más probable encontrar en cada condición conociendo las propiedades de las algas y reconociendo una serie de reglas de ensamblado (Reynolds et al., 2000). Sin embargo, estas predicciones basadas fundamentalmente en la respuesta algal al medio físico (mixis, nutrientes, luz, etc.) pueden ser alteradas por uno de los principales factores de control: la depredación (Harris, 1986; Lampert \& Sommer, 1997).

En este trabajo se ha llevado a cabo un diseño experimental en el que se puede observar la dinámica de un conjunto de algas seleccionadas por sus diferentes tamaños y formas (grandes o pequeñas, coloniales o no, filamentosas o no, móviles o no) y sus diferentes estrategias de crecimiento, en un ambiente sin perturbaciones abióticas (con la excepción de la variación de nutrientes), hasta alcanzar un estado de equilibrio. Este ensayo tiene como objetivos determinar, en condiciones controladas de laboratorio, el establecimiento de asociaciones estables de microalgas como respuesta de las características del grupo funcional al que pertenecen ante ambientes distintos (en cuanto a la concentración de nutrientes y la presencia o no de herbívoros), y analizar los mecanismos implicados en este resultado (competencia, historia del inóculo). También se ha evaluado la influencia de estos dos factores, la concentración de nutrientes y la herbivoría, y su interacción, sobre aspectos estructurales (biomasa total, composición final, diversidad) y funcionales (fotosíntesis y respiración) de la comunidad algal final formada partiendo del mismo conjunto inicial de especies. 
Tabla 1. Grupo taxonómico al que pertenecen y características morfométricas de las especies algales usadas en los experimentos. DEE: Diámetro esférico equivalente. Cél.: célula; Fil.: filamento. Taxonomic group to which they belong and morphometric features of the algal species used in the experiments. DEE: Equivalent Spherical Diameter. Cél.: cell; Fil.: filament.

\begin{tabular}{|c|c|c|c|c|c|}
\hline Especie & Grupo taxonómico & Largo $(\mu \mathrm{m})$ & $\operatorname{Ancho}(\mu \mathrm{m})$ & Biovolumen $\left(\mu \mathbf{m}^{3}\right)$ & DEE $(\mu \mathrm{m})$ \\
\hline Cosmarium contractum & Desmidiácea & $25 \pm 1$ & $32 \pm 4$ & $6833 \pm 1321$ & 50 \\
\hline Cryptomonas ovata & Criptofícea & $16 \pm 1$ & $10 \pm 1$ & $870 \pm 134$ & 23 \\
\hline Euglena gracilis & Euglenofícea & $51 \pm 4$ & $8 \pm 1$ & $1890 \pm 494$ & 33 \\
\hline Limnothrix redekei & Cianofícea & $\begin{array}{c}\text { Cél.: } 5.1 \pm 0.2 \\
\text { Fil.: } 97 \pm 92\end{array}$ & $\begin{array}{c}\text { Cél. y Fil. } \\
1.7 \pm 0.5\end{array}$ & $\begin{array}{c}\text { Cél. } \\
11.6 \pm 0.7\end{array}$ & 16 \\
\hline Monoraphidium contortum & Clorofícea & $32.1 \pm 0.4$ & $2.6 \pm 0.7$ & $57 \pm 2$ & 10 \\
\hline Pediastrum tetras & Clorofícea & Cél: $9 \pm 0.4$ & Alto $=2 \pm 0.2$ & $127 \pm 11$ & 27 \\
\hline Planktothrix agardhii & Cianofícea & $\begin{array}{c}\text { Cél: } 3.1 \pm 0.2 \\
\text { Fil: } 231 \pm 189\end{array}$ & $\begin{array}{c}\text { Cél \& Fil } \\
7.8 \pm 0.4\end{array}$ & $\begin{array}{c}\text { Cél. } \\
148 \pm 12\end{array}$ & 60 \\
\hline Scenedesmus acutus & Clorofícea & $11.7 \pm 2.0$ & $4.0 \pm 0.5$ & $98 \pm 10$ & 19 \\
\hline
\end{tabular}

\section{MATERIAL Y MÉTODOS}

\section{Especies de algas, condiciones de cultivo y tratamientos}

Se han utilizado 8 especies de microalgas cosmopolitas de agua dulce (Tabla 1) para las experiencias de ensamblado de comunidades. Se eligieron representantes de las categorías de unicelulares (móviles o no), coloniales y filamentosas. Dentro de cada categoría se buscaron especies de tamaños más pequeños y más grandes, considerándose pequeñas aquellas algas cuyo diámetro esférico equivalente (DEE) fuera menor de $20 \mu \mathrm{m}$ (nanoplancton) y grandes aquellas cuyo DEE fuera mayor de $20 \mu \mathrm{m}$ (microplancton). Las especies elegidas fueron: Cryptomonas ovata (unicelular, móvil y pequeña), Euglena gracilis (unicelular, móvil y grande), Limnothrix redekei (filamentosa pequeña o fina), Planktothrix agardhii (filamentosa grande o gruesa), Scenedesmus acutus (colonial -4 células/colonia-, inmóvil y pequeña), Pediastrum tetras (colonial -de 8 a 16 células en la mayoría de las colonias, inmóvil y grande), Monoraphidium contortum (unicelular, inmóvil y pequeña) y Cosmarium contractum (unicelular, inmóvil y grande). Las poblaciones de las ocho algas fueron adquiridas en tres centros especializados: la Colección de Cultivos de Algas y Protozoos del Centre for Ecology and Hidrology (CCAP), actualmente sita en la Asociación para las Ciencias Marinas en Escocia (Reino Unido), la Colección de Culti- vos de Algas del Departamento de Botánica de la Universidad de Coimbra (Portugal), y el Max Planck Institute for Limnology (MPIL) en Plön (Alemania). Los cultivos stock se mantenían en matraces de $50 \mathrm{~mL}$ con medio de cultivo Jaworski (CCAP) y fueron estos cultivos stock en fase exponencial de crecimiento los que sirvieron de inóculo para los diferentes experimentos. Los cultivos de estas algas se mantenían en una cámara de cultivo climatizada a $20{ }^{\circ} \mathrm{C}$, con un fotoperiodo de $12 \mathrm{~h}$ luz: $12 \mathrm{~h}$ oscuridad e intensidad luminosa (en radiación fotosintéticamente activa) aproximadamente de $30 \mu \mathrm{E} \cdot \mathrm{m}^{-2} \mathrm{~s}^{-1}$. Estas fueron las condiciones físicas de todas las experiencias realizadas ya que todos los cultivos se dispusieron en la misma cámara.

Para observar la competencia entre algas, se llevaron a cabo cultivos de pares de especies de las dos algas de crecimiento más rápido (Monoraphidium y Scenedesmus) con cada una de las restantes algas. Los inóculos fueron de $250 \mathrm{cel} / \mathrm{ml}$ de densidad final para cada especie de alga. Se utilizaron matraces de $250 \mathrm{ml}$ y un medio de agua mineral enriquecida con $6.2 \mu \mathrm{g} \mathrm{P} / \mathrm{l}$ y $152 \mu \mathrm{gN} / 1$ de concentración final $(\mathrm{N} / \mathrm{P}=25)$, un medio donde la concentración de nutrientes es baja pero que está por encima de los valores que pueden ser limitantes para el crecimiento (Reynolds, 1984). Este medio será denominado a partir de ahora "medio oligotrófico".

Los tratamientos realizados para determinar el efecto "desde arriba", "desde abajo" y su interacción sobre la estructura final y la 
función de la comunidad algal, fueron: (1) medio oligotrófico sin herbívoros, (2) medio eutrófico sin herbívoros, (3) medio oligotrófico con herbívoros y (4) medio eutrófico con herbívoros. Para los experimentos sobre el efecto de los nutrientes en la comunidad algal (todas las algas juntas sin la presencia de herbívoros), se prepararon dos tipos de medio de cultivo: el que hemos designado como oligotrófico y otro con una concentración de $\mathrm{P}$ y N 10 veces superior (62 $\mu \mathrm{g} \mathrm{P} / 1$ y $1520 \mu \mathrm{gN} / 1$ de concentración final) que llamaremos "medio eutrófico". Para comprobar, la viabilidad de las poblaciones algales en su estado final o de senescencia, una alícuota del estado final (tras 40 días de incubación) del cultivo mixto en medio oligotrófico fue resembrada en medio fresco oligotrófico y se siguió el crecimiento de las poblaciones algales durante 20 días. Para averiguar el efecto de los herbívoros sobre la estructuración del ensamblado de microalgas y su función se utilizaron 3 especies de herbívoros: el cladócero Daphnia magna y los rotíferos Keratella cochlearis y Brachionus calicyflorus a razón de 0.5 ind/l de concentración final el primero de ellos y 10 ind/l los rotíferos. Se eligieron estas densidades iniciales pues constituyen valores que pueden darse en medios acuáticos con facilidad. Se añadieron junto a las algas el primer día de experimentación. Se utilizaron los mismos medios de cultivo (oligotrófico y eutrófico), inóculos de los mismos cultivos stock de algas y las mismas condiciones de incubación.

Se tomaban muestras cada 3-5 días durante un mínimo de 36 días y un máximo de 57 días. Todos los tratamientos se realizaron por triplicado. En todos los casos las muestras para recuento algal eran de $2 \mathrm{ml}$ y se fijaban inmediatamente con lugol para su posterior recuento tras sedimentación en cámaras Utermöhl y observación mediante un microscopio invertido. La densidad algal se obtuvo a partir del recuento de al menos 400 células de cada población. La densidad algal se transformó en biovolumen multiplicando por el valor del volumen celular de cada especie de alga (Tabla 1). El biovolumen celular se calculó a partir de las dimensiones de las distintas especies de algas, aproximando sus formas a las figuras geométricas más parecidas (Rott, 1981). Para obtener la biomasa algal en peso fresco se consideró una densidad igual a $1 \mathrm{~g} / \mathrm{cm}^{3}$. Las tasas intrínsecas de crecimiento $(r)$ de las microalgas a los 10 días de iniciados todos los cultivos se calcularon suponiendo un modelo exponencial de crecimiento. Para el recuento de los herbívoros de pequeño tamaño se filtraban $50 \mathrm{ml}$ de cultivo por una malla de nytal de $37 \mu \mathrm{m}$ de poro cada 510 días y se devolvían al medio. Los ejemplares de Daphnia fueron recontados de visu.

\section{Medidas de actividad metabólica: fotosíntesis y respiración}

Las tasas de fotosíntesis y respiración de la comunidad algal se determinaron en los cultivos con y sin presencia de herbívoros. Para ello se utilizó un electrodo de Clark (Hansatech, Dubinsky et al., 1987), el cual detecta cambios de voltaje debidos a las diferentes concentraciones de oxígeno disuelto en el agua. Se filtraba una cierta cantidad de cultivo algal (entre 20 y $250 \mathrm{ml}$, dependiendo de la densidad algal del cultivo). Las células recogidas en el filtro eran resuspendidas en $2 \mathrm{ml}$ del filtrado. En el caso de los tratamientos con herbívoros, si tras el filtrado se observaba algún ejemplar de Daphnia, éste era retirado del filtro antes de resuspender el material depositado; la respiración de los rotíferos no es detectable en estas condiciones de medida (dato no publicado). Las muestras de los cultivos se sometían a una radiación fotosintéticamente activa de 100 $\mu \mathrm{E} \cdot \mathrm{m}^{-2} \cdot \mathrm{s}^{-1}$ para medir la producción de oxígeno por fotosíntesis. Esta intensidad luminosa no es saturante (Carignan et al., 2000) y por otro lado es suficientemente elevada para determinar cambios en la concentración de oxígeno debidos a fotosíntesis en cortos períodos de tiempo. Posteriormente, la misma muestra era sometida a oscuridad para registrar la disminución de oxígeno en el cultivo algal debido a su consumo por respiración. Una curva de calibrado nos permitió transformar el voltaje en $\mathrm{mgO}_{2} \cdot \mathrm{l}^{-1} \mathrm{~h}^{-1}$ producidos y/o consumidos. La producción primaria neta (PPN) y la respiración se expresaron por unidad de peso seco de materia algal. El peso seco se 
consideró que era el $10 \%$ del peso fresco. Se transformó la producción de oxígeno en carbono utilizando la ratio de conversión (peso a peso) $\mathrm{C}: \mathrm{O}_{2}$ de 0.312 (Vollenweider, 1969). Las tasas de fotosíntesis y respiración se determinaron en 6-10 ocasiones para cada cultivo repartidas a lo largo del tiempo de cultivo.

\section{Métodos estadísticos}

Para detectar diferencias en el crecimiento de las dos algas de mayor crecimiento, Scenedesmus y Monoraphidium, en competencia con cada una de las 7 restantes algas se realizaron sendos análisis de la varianza (ANOVA) de una vía con los datos de la densidad celular de estas dos algas a los 19 días de los cultivos. Se realizaron ANOVAs de dos vías siendo los factores la Concentración de Nutrientes y la Herbivoría y las variables a comparar la biomasa total algal, la biomasa de cada población algal (todas ellas promediadas hasta los 36 días de cultivo) y su tasa intrínseca de crecimiento (calculada a los 10 días de cultivo), así como sobre la producción primaria neta y la respiración (también promediadas hasta los 36 días). La homogeneidad de varianzas se comprobó mediante el test de Levene. Las pruebas post hoc de Bonferroni se llevaron a cabo para distinguir entre medias di- ferentes que permitieran agrupar conjuntos de tratamientos con una $p<0.05$. Todos los análisis estadísticos se realizaron con el paquete estadístico SPSS versión 13.0.

\section{RESULTADOS}

\section{Factores de control del ensamblado de microalgas}

Las tasas de crecimiento $(r)$ de las diferentes poblaciones algales en los primeros 10 días de cultivo (Tabla 2), que es cuando los cultivos resembrados en medio fresco experimentan su fase exponencial de crecimiento, indican que Scenedesmus y Monoraphidium crecen más rápidamente en prácticamente todos los tratamientos, con tasas máximas en situación de eutrofia sin depredación (Tabla 2). Cryptomonas y Pediastrum presentan sus mayores $r$ en situación de eutrofia con depredadores. Por el contrario Limnothrix parece que se ve favorecida por las situaciones de oligotrofia y Cosmarium por la situación de mayor estrés (oligotrofia con depredación). Euglena y Planktothrix presentaron las $r$ más bajas, siendo incluso negativas cuando los herbívoros estaban presentes y ausentes, respectivamente. El análisis de la varianza de dos vías (concentración de nutrientes y herbivoría) sobre las tasas de creci-

Tabla 2. Tasas intrínsecas de crecimiento $\left(r\right.$, días $\left.^{-1}\right)$ de las ocho especies de algas para los 10 primeros días de cultivo (suponiendo un modelo de crecimiento exponencial) calculadas sobre los datos de densidad celular en los distintos tratamientos de ensamblado de comunidades. Se indican las medias y errores típicos (entre paréntesis). Mn: Monoraphidium contortum; Sc: Scenedesmus acutus; Cr: Cryptomonas ovata; Pe: Pediastrum tetras; Co: Cosmarium contractum; Eu: Euglena gracilis; Li: Limnothrix redekei; Pl: Planktothrix agardhii. Intrinsic growth rates $\left(r\right.$, days $\left.{ }^{-1}\right)$ of the eight algae species for the first 10 days of culture (assuming an exponential growth model) calculated on cell density data in the different treatments of community ensembles. Means and standard errors (in brackets) are indicated.

\begin{tabular}{lcccccccc}
\hline Tratamiento & $\mathrm{Mn}$ & $\mathrm{Sc}$ & $\mathrm{Cr}$ & $\mathrm{Pe}$ & $\mathrm{Co}$ & $\mathrm{Li}$ & $\mathrm{Eu}$ & $\mathrm{Pl}$ \\
\hline $\begin{array}{l}\text { Oligotrófico SIN } \\
\text { depredación }\end{array}$ & 0.45 & 0.53 & 0.26 & 0.32 & 0.03 & 0.45 & 0.02 & -0.78 \\
& $(0.01)$ & $(0.02)$ & $(0.02)$ & $(0.01)$ & $(0.03)$ & $(0.02)$ & $(0.01)$ & $(0.00)$ \\
$\begin{array}{l}\text { Eutrófico SIN } \\
\text { depredación }\end{array}$ & 0.63 & 0.74 & 0.17 & 0.17 & 0.09 & 0.07 & 0.00 & -0.71 \\
$\begin{array}{l}\text { Oligotrófico CON } \\
\text { depredación }\end{array}$ & $(0.04)$ & $(0.01)$ & $(0.18)$ & $(0.04)$ & $(0.03)$ & $(0.10)$ & $(0.02)$ & $(0.00)$ \\
$\begin{array}{l}\text { Eutrófico CON } \\
\text { depredación }\end{array}$ & 0.39 & 0.35 & 0.19 & 0.06 & 0.15 & 0.26 & -0.10 & 0.03 \\
& $(0.08)$ & $(0.07)$ & $(0.03)$ & $(0.07)$ & $(0.02)$ & $(0.02)$ & $(0.06)$ & $(0.09)$ \\
& $(0.04)$ & $(0.08)$ & $(0.01)$ & $(0.01)$ & $(0.01)$ & $(0.10)$ & $(0.02)$ & $(0.02)$ \\
\hline
\end{tabular}




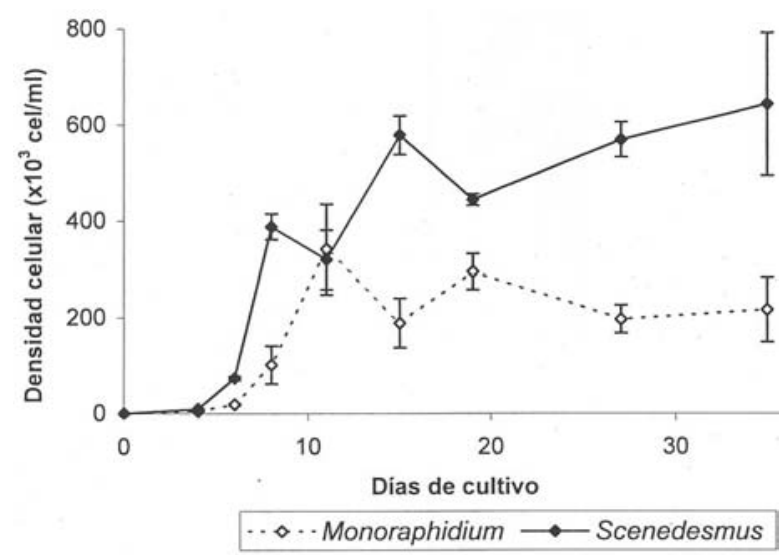

Figura 1. Variación de la densidad celular de Monoraphidum y Scenedesmus en un cultivo con medio oligotrófico enfrentando este par de especies. Variation of Monoraphidum and Scenedesmus cell density in competition in an oligotrophic culture medium.

miento (Tabla 3) reveló que dicha tasa para Monoraphidium y Scenedesmus en los primeros días del ensamblado no se ve afectada por la concentración de nutrientes, en cambio sí hay una reducción significativa en presencia de herbívoros. La tasa de crecimiento de Cryptomonas no es significativamente diferente entre tratamientos y Pediastrum sí mostró una diferencia significativa de su $r$ debida a los nutrientes y a la interacción de factores. Cosmarium presentó tasas de crecimiento estadísticamente diferentes solo en función de la presencia de herbívoros y de la interacción de factores. La $r$ de Limnothrix solo es significativamente diferente debido a los nutrientes y en el caso de Euglena y Planktothrix, sólo debido a los herbívoros.

Para analizar con mayor detalle la capacidad de crecimiento conjunto de las dos algas con tasas de crecimiento más altas, Scenedesmus y Monoraphidium, se llevó a cabo un cultivo oligotrófico con ambas inoculadas a la vez en igual densidad inicial (Fig. 1). Scenedesmus se reveló como el competidor más eficaz, alcanzando tasas de crecimiento más altas y densidades más elevadas. Para confirmar la mayor capacidad competitiva de las especies Scenedesmus y Monoraphidium con el resto de algas del estudio, se realizaron cultivos por pares de especies de cada una de éstas por separado con el resto de las
Tabla 3. Resultados del ANOVA de dos vías con el contenido en nutrientes y la presencia o no de herbívoros como factores sobre la tasa intrínseca de crecimiento de cada una de las especies algales (significación $p<0.05$ ). ns: no significativo. g.l.: grados de libertad. Abreviaturas de las algas como en Tabla 2. Two-way ANOVA results with nutrient concentration and the presence or absence of herbivores as factors on intrinsic growth rates of each algal species (significance $p<0.05$ ). ns: non significant; g.l: degrees of freedom. Abbreviations for algae as in Table 2.

\begin{tabular}{|c|c|c|c|c|c|c|c|c|c|}
\hline Fuente & g.l. & Mn & Sc & $\mathbf{C r}$ & Pe & Co & $\mathbf{L i}$ & Eu & Pl \\
\hline Nutri & 1 & ns & ns & ns & 0.004 & ns & 0.002 & ns & ns \\
\hline Herbívoros & 1 & 0.035 & 0.010 & $n s$ & ns & 0.026 & ns & 0.020 & 0.000 \\
\hline Nutrientes $\times$ & & & & & & & & & \\
\hline Herbívoros & 1 & ns & ns & ns & 0.000 & 0.026 & ns & ns & ns \\
\hline
\end{tabular}

algas, en medio oligotrófico (Fig. 2). En todos los casos ambas algas crecían más rápidamente y alcanzaban densidades poblacionales superiores al resto de las especies. También se pudo constatar que las densidades finales alcanzadas por una especie quedaban determinadas por la identidad de la otra especie competidora. Así, en el caso de Monoraphidium (Fig. 2), este alga alcanzó densidades mínimas cuando crecía en un cultivo mixto con Scenedesmus y máximas cuando crecía en competencia con las algas de crecimiento lento como Euglena, Cosmarium y Planktothrix. Es interesante resaltar la similitud de su desarrollo en presencia de Cryptomonas y Pediastrum ya que estas dos algas son de morfología bien diferente. Un ANOVA de una vía, sobre la densidad de Monoraphidium alcanzada a los 19 días de cultivo (tomado este día como

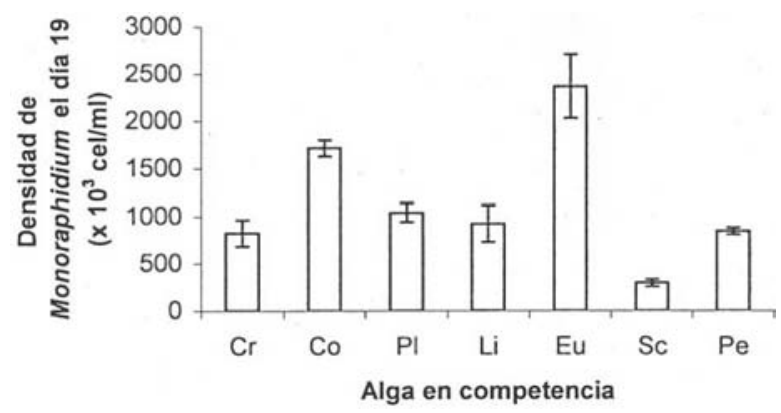

Figura 2. Densidad celular de Monoraphidium alcanzada a los 19 días en los cultivos de medio oligotrófico para estudiar la competencia a pares de especies. Abreviaturas de las especies como en Tabla 2. Monoraphidum cell density reached at day 19 in oligotrophic cultures to study competition with the remaining seven algae. Abbreviations for species as in Table 2. 
representativo de la dinámica de crecimiento) con cada una de las algas restantes, reveló que dicha densidad era distinta en función del alga competidora y lo mismo ocurrió con la densidad de Scenedesmus en cultivo mixto con cada una de las otras algas (en ambos casos $p<0.000$ ).

Al observar las dinámicas de la biomasa de cada una de las poblaciones algales en los dife- rentes tratamientos (Fig. 3) se aprecia una pauta similar tanto en Monoraphidium como en Scenedesmus (Fig. 3A y B). El ANOVA de dos vías sobre la biomasa promedio (Tabla 4) indica que tanto el factor Nutrientes como el factor Herbivoría, así como su interacción, generan diferencias estadísticamente significativas en la biomasa de estas dos especies. La tendencia
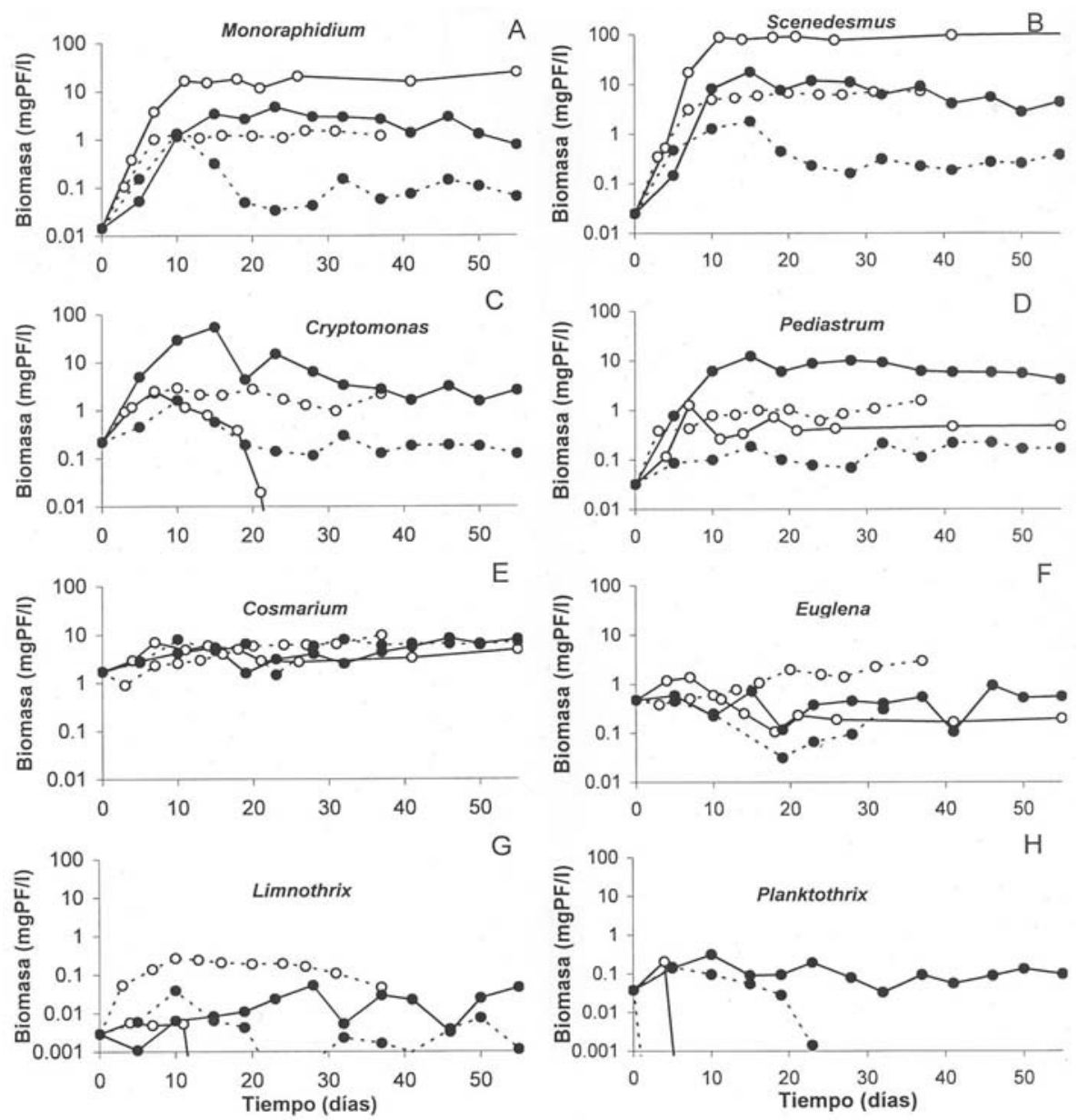

$$
\begin{aligned}
& \text {... - Oligotrófico SIN depredación } \\
& \text {.- - - Oligotrófico CON depredación }
\end{aligned}
$$

Figura 3. Variación de la biomasa de las diferentes especies de algas a lo largo del tiempo en los distintos tipos de medios de cultivo (tratamientos). Las líneas discontinuas indican medio oligotrófico y las líneas continuas medio eutrófico. Los símbolos blancos indican la ausencia de herbívoros, los símbolos negros indican la presencia de herbívoros. Nótese la escala logarítmica en el eje de ordenadas. Cada punto corresponde al promedio de tres réplicas (no se muestran las barras de error para facilitar la observación de las figuras). Variation of algal species biomass throughout time in the different culture media (treatments). Broken lines indicate oligotrophic and solid lines indicate eutrophic media respectively. White symbols indicate absence and black symbols indicate presence of herbivores respectively. Notice the logarithmic scale in ordinate axis. Each point corresponds to the average of three replicates (error bars are not shown for clarity of figures). 
de Cryptomonas y Pediastrum fue similar entre ellas (Fig. 3C y D): ambas, y a diferencia de las dos anteriores, presentaron valores de biomasa máximos en el medio eutrófico con depredación. Los resultados del ANOVA de dos vías para estas especies fueron semejantes a los de Monoraphidium y Scenedesmus (Tabla 4). Cosmarium (Fig. 3E) no mostró grandes variaciones de biomasa entre los distintos tratamientos y no hubo diferencias estadísticamente significativas debido a los tratamientos (Tabla 4). Euglena (Fig. 3F) que alcanzó una biomasa no demasiado elevada, no superando el valor de $3 \mathrm{mgPF} / \mathrm{l}$, mostró diferencias estadísticamente significativas dependiendo de cada uno de los factores ensayados así como de su interacción (Tabla 4) y alcanzó una mayor biomasa en el medio oligotrófico sin depredación. Del mismo modo, la biomasa alcanzada por las dos cianofíceas filamentosas (Limnothrix y Planktothrix, Fig. 3G y $3 \mathrm{H}$ ) fue muy baja (siempre por debajo de $1 \mathrm{mgPF} / \mathrm{l})$. Planktothrix, al igual que Cosmarium, no presentó diferencias entre tratamientos. Limnothrix presentó diferencias estadísticamente significativas en su biomasa en función de la cantidad de nutrientes y la presencia o no de herbívoros, presentado una mayor biomasa en el medio oligotrófico sin depredación como ocurría con Euglena.

La biomasa de los herbívoros también fue marcadamente diferente entre tratamientos. Siendo idéntica la densidad de herbívoros inoculada en cada réplica, se alcanzaron en promedio densidades muy distintas en cada tratamiento. Así,

Tabla 4. Resultados del ANOVA de dos vías con el contenido en nutrientes y la presencia o no de herbívoros como factores para la biomasa de cada una de las especies algales promediada hasta el día 36 de cultivo (significación $p<0.05$ ). Abreviaturas de las algas y símbolos como en Tablas 2 y 3. Two-way ANOVA results with nutrient concentration and the presence or absence of herbivores as factors on biomass of each algal species averaged until culture day 36 (significance $p<0.05$ ). Abbreviations for algae and symbols as in Tables 2 and 3.

\begin{tabular}{lccccccccc}
\hline Fuente & g.l. & Mn & Sc & Cr & Pe & Co & Li & Eu & PI \\
\hline Nutrientes & 1 & 0.000 & 0.000 & 0.011 & 0.002 & $\mathrm{~ns}$ & 0.002 & 0.001 & $\mathrm{~ns}$ \\
Herbívoros & 1 & 0.000 & 0.000 & 0.014 & 0.003 & $\mathrm{~ns}$ & 0.002 & 0.000 & $\mathrm{~ns}$ \\
Nutrientes $\times$ & & & & & & & & & \\
Herbívoros & 1 & 0.000 & 0.000 & 0.005 & 0.001 & $\mathrm{~ns}$ & 0.001 & 0.000 & $\mathrm{~ns}$ \\
\hline
\end{tabular}

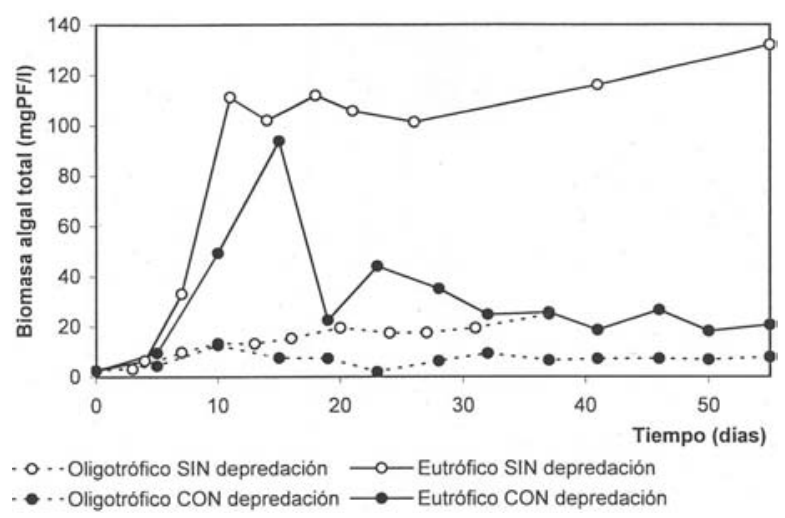

Figura 4. Variación de la biomasa total de la comunidad algal a lo largo del tiempo en los distintos tipos de medios de cultivo (tratamientos). Misma simbología que en la figura 3. Cada punto corresponde al promedio de tres réplicas (no se muestran las barras de error para facilitar la observación de la gráfica). Variation of total algal biomass throughout the course of time in the different culture media (treatments). Same symbols as in Figure 3. Each point corresponds to the average from three replicates. (error bars are not shown for clarity of figures).

en medio oligotrófico Brachionus presentó una densidad media de $2 \pm 0$ ind/l, Keratella de 2 \pm 1 ind/l y Daphnia de $11 \pm 2$ ind/l; en medio eutrófico Brachionus alcanzó $84 \pm 80$ ind/l, Keratella $10 \pm 4$ ind/l y Daphnia $18 \pm 1$ ind/l.

La biomasa total alcanzada por la comunidad algal en cada tratamiento es diferente como resultado de la diferente dinámica de las algas que componen el ensamblado. La biomasa de la comunidad algal en el medio eutrófico sin la presencia de herbívoros fue la más elevada de todos los tratamientos ensayados (Figs. 4 y 5). Los valores máximos (111-130 mgPF/l) en este cultivo se alcanzaron a partir del día 10 y desde ese momento se mantuvo casi constante alrededor de estas cifras. En el medio eutrófico que contenía los herbívoros la biomasa algal aumentó más lentamente hasta el día 15 de cultivo (máximo valor $94 \mathrm{mgPF} / \mathrm{l}$ ) y a partir de entonces se observó una importante reducción debido al consumo de algas por parte de los herbívoros que la redujo a valores de $22 \mathrm{mgPF} / 1$. La biomasa total de algas en medio oligotrófico con y sin depredación mostró valores semejantes hasta el día 10 de cultivo, momento en el que alcanzó valores de $13 \mathrm{mgPF} / \mathrm{l}$, cifra que se man- 
Tabla 5. Resultados del ANOVA de dos vías con el contenido en nutrientes y la presencia o no de herbívoros como factores para la biomasa total algal, la producción primaria neta (PPN) y la respiración promediadas hasta el día 36 de cultivo (significación $p<0.05$ ). Símbolos como en Tabla 3. Table 5. Two-way ANOVA results with nutrient concentration and the presence or absence of herbivores as factors on total algal biomass, net primary production and respiration averaged until culture day 36 (significance $p<0.05$ ). Symbols as in Table 3.

\begin{tabular}{lcccc}
\hline Tratamiento & g.l. & Biomasa total & PPN & Respiración \\
\hline Nutrientes & 1 & 0.000 & 0.000 & 0.000 \\
Herbívoros & 1 & 0.000 & 0.004 & $\mathrm{~ns}$ \\
Nutrientes $\times$ & & & & \\
Herbívoros & 1 & 0.000 & $\mathrm{~ns}$ & 0.011 \\
\hline
\end{tabular}

tuvo en el cultivo sin herbívoros y que se redujo ligeramente en presencia de éstos (Fig. 4).

La biomasa total algal en medio eutrófico sin depredadores es unas 5 veces mayor que en medio oligotrófico (Fig. 5), cuando hay herbívoros en medio eutrófico la biomasa también es aproximadamente 5 veces superior a la alcanzada en medio oligotrófico con herbívoros. Además, la presencia de herbívoros reduce a la mitad la biomasa promedio alcanzada tanto en el sistema oligotrófico como en el eutrófico. La variabilidad, dentro de cada tratamiento, de la biomasa algal resultó estar relacionada con los factores de control, de forma que cuando no estaban presentes los depredadores tanto en oligotrofia como en eutrofia, el coeficiente de variación $(\mathrm{CV})$ de la biomasa alcanzada por las tres réplicas fue del $9 \%$ y cuando existen depredadores se aumenta la varia-

Tabla 6. Resultados de la prueba post hoc de Bonferroni del análisis de la varianza de una vía. Sólo se indican las probabilidades significativas ( $p<0.05$, ns: no significativo). En los tratamientos, "SIN" indica la ausencia de herbívoros en el medio y "CON" la presencia de éstos. Results of post hoc Bonferroni post-hoc test of one-way ANOVA. Only significant probabilities are shown $(p<0.05 ;$ ns: non significant). In treatments, "SIN" indicates the absence and "CON" the presence of herbivores respectively.

\begin{tabular}{lc}
\hline \multicolumn{1}{c}{ Tratamiento } & Biomasa total \\
\hline Oligotrófico SIN/Oligotrófico CON & $\mathrm{ns}$ \\
Oligotrófico SIN/Eutrófico SIN & 0.000 \\
Oligotrófico SIN/Eutrófico CON & 0.010 \\
Eutrófico SIN/Oligotrófico CON & 0.000 \\
Eutrófico SIN/Eutrófico CON & 0.000 \\
Oligotrófico CON/Eutrófico CON & 0.000 \\
\hline
\end{tabular}

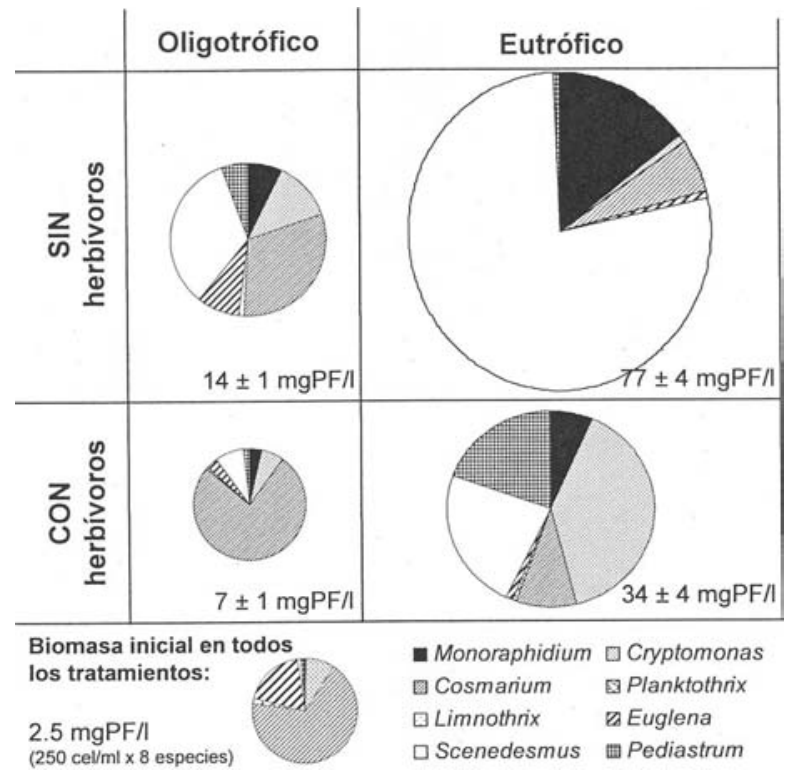

Figura 5. Proporción de la biomasa (media de las tres réplicas y hasta el día 36 de cultivo) alcanzada por las diferentes especies de algas en los distintos tratamientos. El distinto tamaño de las gráficas intenta representar los diferentes valores de biomasa total, la cual se encuentra indicada en los números adjuntos (media \pm error típico). Se muestra también la biomasa algal inicial (y el reparto por especies) que fue idéntica para todos los tratamientos. Proportion of biomass (averaged for three replicates and until culture day 36) reached by each algal species in the treatments. The different sizes of the graphs roughly represent the different total biomass values, which are also indicated (mean of three replicates \pm standard error). Total initial biomass (the same in all treatments) and species contribution are also indicated.

bilidad del resultado hasta un CV del $20 \%$ para ambos estados tróficos.

Un ANOVA de dos vías, con el contenido en nutrientes y la presencia/ausencia de depredadores como factores, sobre la biomasa algal promediada para los 36 días de cultivo, reveló que había diferencias estadísticamente significativas debidas tanto a la concentración de nutrientes en el medio como a la presencia o no de depredadores (Tabla 5). Además, la interacción de estos dos factores también fue significativa. La prueba post hoc de Bonferroni nos permite observar agrupaciones de tratamientos de manera que en base a la biomasa total se puede decir que (Tabla 6) se diferencia significativamente la biomasa alcanzada en medio eutrófico o en oligotrófico, cuando el 


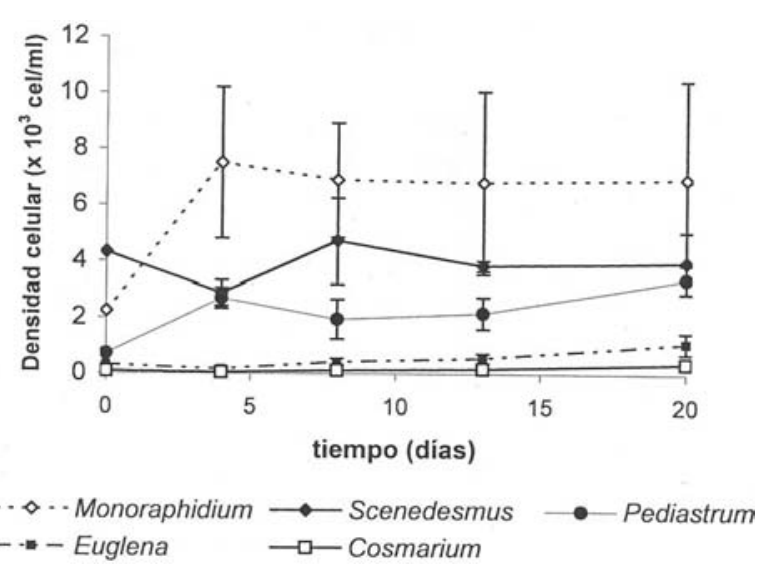

Figura 6. Densidades celulares alcanzadas por las algas tras ser resembradas en medio fresco oligotrófico a partir de un cultivo mixto de las 8 algas de 40 días. Cell densities of algal species after reinoculation in new oligotrophic medium from a 40-day old mixed culture.

medio es oligotrófico la presencia de herbívoros no altera significativamente la biomasa, lo que sí ocurre cuando el medio es eutrófico.

Por último, y para comprender otro factor ligado a la estrategia de permanencia de una $\mathrm{u}$ otra especie en el ensamblado, se estudió la capa- cidad de recuperación poblacional de cada alga (Fig. 6), al inocular en medio fresco oligotrófico una alícuota de un cultivo mixto de las 8 algas de 40 días. Se observó que Monoraphidium alcanzó las densidades más altas, superiores incluso a Scenedesmus. Cosmarium, Pediastrum y Euglena, todas ellas de crecimiento lento, parece que perduran e incluso comienzan a crecer en el nuevo medio. En cambio, Planktothrix, Limnothrix y Cryptomonas no se desarrollaron en este nuevo cultivo.

\section{Actividad metabólica de la comunidad algal en los diferentes tratamientos}

Los valores máximos de producción primaria bruta (normalizada para la biomasa, PPB) alcanzados en los diferentes tratamientos fueron de $0.02 \mathrm{mgC} \cdot \mathrm{mgPS}^{-1} \cdot \mathrm{h}^{-1}$ en el medio oligotrófico con depredación, de $0.04 \mathrm{mgC} \cdot \mathrm{mgPS}^{-1} \cdot \mathrm{h}^{-1}$ tanto en el medio oligotrófico sin depredación como en el medio eutrófico con depredación y 0.12 $\mathrm{mgC} \cdot \mathrm{mgPS}^{-1} \cdot \mathrm{h}^{-1}$ en el medio eutrófico sin herbívoros. En todos los tratamientos, la pro-
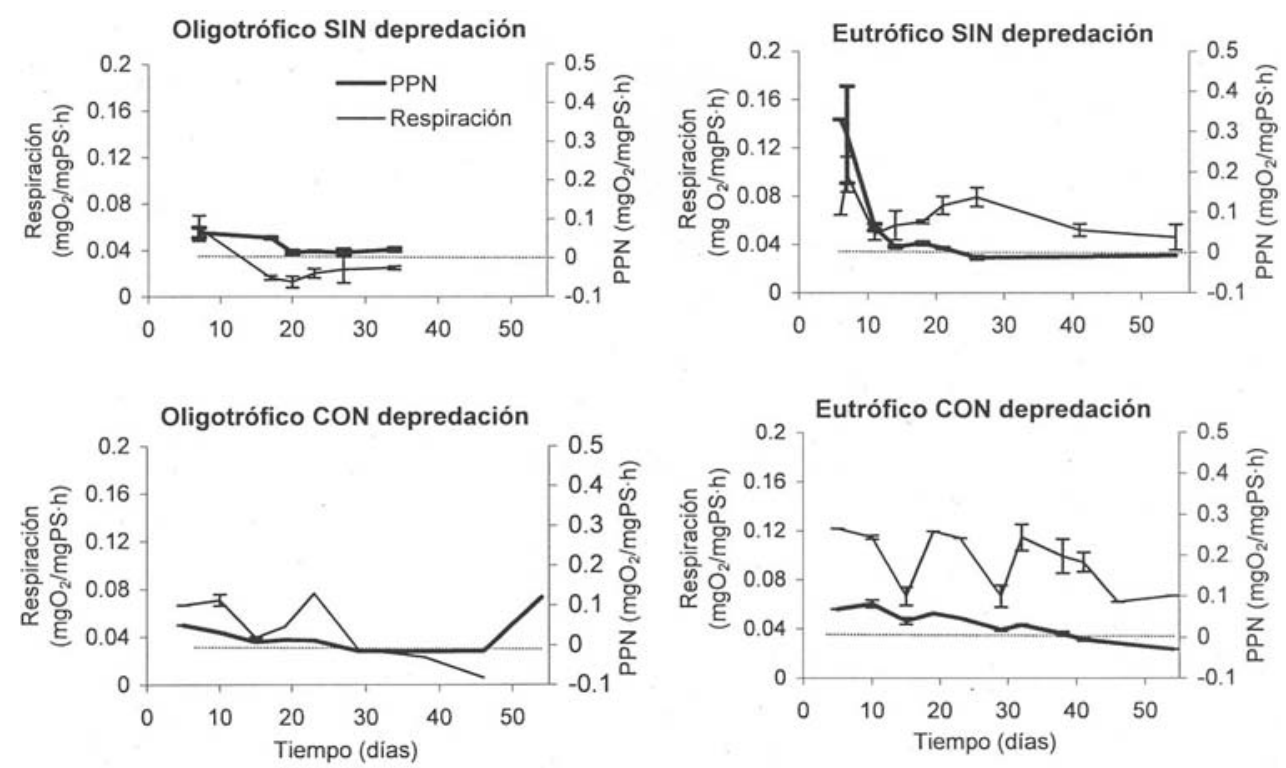

Figura 7. Variación de la producción primaria neta (PPN, línea gruesa) y de la respiración (línea fina) (media de tres réplicas y error típico) a lo largo del tiempo de la comunidad algal en los diferentes tratamientos. La línea horizontal indica el 0 en el eje de ordenadas que representa la PPN. Variation of net primary production (PPN, thick line) and respiration (thin line) (average of three replicates and standard error) throughout the time of the algal community in the different treatments. The dotted horizontal line indicates 0 in the ordinate axis (PPN). 

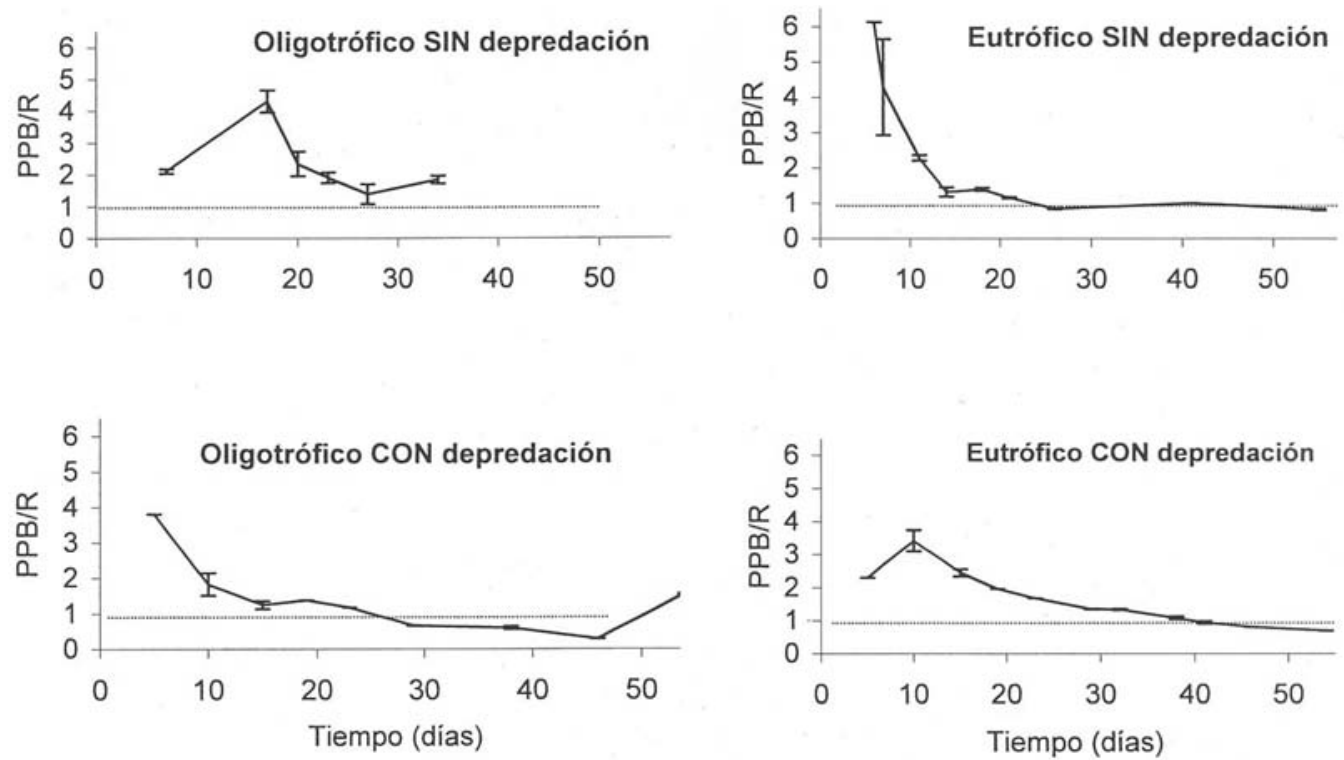

Figura 8. Variación de la relación entre producción primaria bruta (PPB) y respiración (media de tres réplicas y error típico) a lo largo del tiempo de la comunidad algal en los diferentes tratamientos. La línea horizontal indica la divisoria entre la autotrofia neta y la heterotrofia neta. Variation of gross primary production/respiration ratio (average of three replicates and standard error) throughout time, of the algal community in the different treatments. The dotted horizontal line indicates the boundary between net autotrophy and net heterotrophy.

ducción primaria neta por unidad de biomasa (PPN) fue más elevada los primeros días de cultivo y disminuyó con el tiempo hasta alcanzar valores ligeramente negativos en algunos casos (Fig. 7). La PPN de los primeros días de cultivo fue más baja en los tratamientos de medio oligotrófico sin y con la presencia de herbívoros y en el tratamiento eutrófico con depredación. En cambio, en el tratamiento de medio eutrófico sin depredación la producción fotosintética fue marcadamente superior. Un análisis de la varianza, con la concentración de nutrientes y la presencia o no de herbívoros como factores, indicó que había diferencias estadísticamente significativas en la PPN, promediada hasta el día 36 de cultivo (duración del tratamiento más corto), debidas tanto a la concentración de nutrientes como a la presencia o no de depredadores (Tabla 5). La comunidad algal desarrollada en el medio eutrófico sin depredación, que estaba caracterizada por un claro predominio de las algas de pequeño tamaño y crecimiento rápido (Scenedesmus y Monoraphidium), presentó niveles de fo- tosíntesis (normalizada para la biomasa) significativamente superiores (hasta 4 veces más) a las del ensamblado algal de medio oligotrófico, este último caracterizado por una mayor dominancia de especies de gran tamaño y crecimiento lento como Cosmarium y Euglena.

La respiración de la comunidad algal mostró notables diferencias entre los distintos tratamientos (Fig. 7). La respiración fue mínima en el tratamiento de medio oligotrófico sin presencia de herbívoros y máxima en el tratamiento de medio eutrófico con depredación, aunque en este último caso la dinámica de la respiración fue bastante fluctuante. El ANOVA (Nutrientes y Herbivoría como factores) sobre los valores de respiración promediados hasta el día 36, muestra que hay diferencias significativas en función del contenido en nutrientes pero no de la presencia o ausencia de herbívoros en el medio (Tabla 5). La actividad respiratoria, la cual fue significativamente mayor (de dos a seis veces) en el medio eutrófico sin depredación que en el oligotrófico, está también 
relacionada con las diferencias en la composición algal de los distintos ensamblados alcanzados en los tratamientos.

La relación producción primaria bruta/respiración (PPB/R, Fig. 8) varió entre mínimos de 0.6 y máximos de 6.1 , y no en todos los tratamientos fue máxima los primeros días de cultivo. En el medio oligotrófico sin depredación fue máxima hacia el día 17 de cultivo y en el tratamiento de medio eutrófico con presencia de herbívoros lo fue el día 10.

\section{DISCUSIÓN}

En este ensamblado de especies algales, donde han podido tener lugar interacciones como la competencia y la depredación, se observa como los factores de control actúan modificando la biomasa total alcanzada porque se seleccionan determinadas combinaciones de especies. De manera que, como cabría esperar, en un medio más eutrófico la biomasa algal total alcanzada es mayor que en medio oligotrófico y en presencia de herbívoros se ve reducida la biomasa de productores primarios (Vollenweider, 1969; Cottingham et al., 2004). Pero, lo que resulta relevante destacar es la variabilidad de la respuesta, de modo que, sin presencia de herbívoros, la biomasa total alcanzada y la composición del ensamblado resulta muy estable tanto en oligotrofia como en eutrofia, es decir tanto en comunidades con prácticamente un monocultivo de una especie oportunista como en aquellas de gran diversidad, incluso de mayor diversidad (en términos de biomasa) que en el origen. Sin embargo, cuando la presencia de las microalgas es reducida o controlada por los herbívoros, se observa que su variabilidad es mayor y tampoco está relacionada con la diversidad o estructura alcanzada. Por tanto, ante un conjunto de especies de diferente función encontraremos un grado de predictibilidad independiente de la diversidad y controlado por la herbivoría. Y por tanto, no sólo la biomasa total es alterada cuando hay herbívoros, como cabe esperar, sino también la composición de la asociación de algas y con ello la diversidad y su estabilidad o capacidad de presentar, dentro de unos limites de biomasa, una gran variedad de comunidades diferentes. Todo ello se ha visto en un experimento de laboratorio donde el número de variables involucradas es bajo. Así en condiciones naturales, donde otros factores pueden estar influyendo (biogeoquímicos y físicos como la turbulencia, estrés lumínico, etc.), todavía cabría esperar una mayor variabilidad en las comunidades.

Las diferentes estructuras de la comunidad algal que suponen el estado final (steady state) de cada experiencia son el resultado de cómo los factores de control afectan de muy diversa manera, no sólo a las diferentes algas sino también a las diferentes combinaciones de las mismas. Además, la caracterización de las algas en función de su estrategia de crecimiento, no es la única información útil (no es determinante o concluyente) a la hora de predecir cuál será el ensamblado de algas en el transcurso del tiempo. La interacción combinada de factores generará diferentes estados alternativos finales. Así por ejemplo, la mayor concentración de nutrientes favoreció la proliferación de las especies algales de pequeño tamaño y crecimiento rápido como es el caso principalmente de Scenedesmus y en menor medida de Monoraphidium (especies colonizadoras, Olrik, 1994; Reynolds, 1997). En cambio, la situación es muy distinta si a pesar de crecer las algas en un medio eutrófico están los herbívoros ejerciendo su presión de depredación ya que estas especies son muy susceptibles a la depredación por no ser móviles y de pequeño tamaño (Olrik, 1984; Reynolds, 1984). Pero además, el resultado de estos dos factores (enriquecimiento y depredación) se verá modificado la composición de especies de algas que se dan en cada caso, de modo que, por ejemplo, en un medio eutrófico con depredadores estas dos algas oportunistas (Scenedesmus y Monoraphidium) no sólo tendrán que resolver el compromiso entre mayor crecimiento y mayor depredación sino que además se verán en un ensamblado con Pediastrum y Cryptomonas, las cuales son especies fuertemente competidoras. En el caso opuesto encontramos aquellas algas de crecimiento lento y poco atractivas para los depredadores como son Cosmarium, Euglena y Planktothrix. La presencia o ausencia de estas especies en las comunidades finales es 
el resultado de sus tasas de crecimiento iniciales que no les permiten incrementar su presencia ante las colonizadoras, pero además, de su capacidad de mantenerse frente a la presión de herbivoría. Así, a Cosmarium y a Planktothrix principalmente no les afecta la herbivoría, a la primera más por razón de su alta tasa de sedimentación que por su tamaño y a la segunda por su tamaño y características morfológicas (Gliwicz, 1990). De modo que así como las especies colonizadoras tienen igual comportamiento ante los dos factores (nutrientes y depredación), las ruderales (grandes de crecimiento lento) exhiben muy diferente mortalidad causada por los herbívoros. Esto hará que en aquellas condiciones donde sea mayor su presencia relativa, debida a una menor eficacia de las oportunistas, la variabilidad de respuesta sea mayor frente a los herbívoros y por tanto también la interacción o combinación de ellas, produciendo un efecto de mayor diversidad y mayor inestabilidad entendida como falta de predictibilidad.

Otro aspecto fundamental en la consecución de uno $u$ otro estado final es la historia del inóculo (Pádisak, 1992), y como hemos podido observar tras una situación que provoque un estado de senescencia de las poblaciones, como puede ser un largo periodo de estabilidad y falta de recursos (Sommer et al., 1986), la viabilidad de los inóculos de cada población en declive será diferente. De modo que, por ejemplo, la población de crecimiento mas rápido (Scenedesmus) y claramente dominante en competencia con la otra colonizadora (Monoraphidium), no es la que tiene un inóculo más eficaz. Esto es lo que puede producir una alternancia entre ellas como dominantes en condiciones muy similares. Otro claro ejemplo es el de Cryptomonas y Pediastrum, que como ya se ha visto, se han comportado, pese a su muy diferente morfología, como unidades funcionales semejantes en todos los tratamientos y así las considera el modelo empírico de campo PEG (Sommer et al., 1986). Estas algas son eficaces oportunistas que se ven favorecidas por la relajación de la competencia con Scenedesmus y Monoraphidium gracias a la gran presión del herbívoro sobre estas últimas. Sin embargo, se diferencian en que Pediastrum, en virtud de su polimorfismo, presenta grandes colonias que le permiten sobrevivir al estrés de la depredación (Rojo et al., 2000) y por eso, aunque tanto Olrik (1994) como Reynolds (1997) lo consideran un posible colonizador (C-Olrik, 1994, Association J Reynolds, 1997) también se considera este género como S (tolerante al estrés, Olrik, 1994). Y además, presenta una alta viabilidad de su población senescente ante nuevas circunstancias lo que puede hacer que en la historia de un ensamblado llegue a ser alterna con Cryptomonas, de inóculo senescente menos viable.

En el año 2000, un debate entre especialistas en ecología del fitoplancton (Reynolds et al., 2000) culminó con 10 reglas de ensamblado (en el sentido de Weiher \& Keddy, 1999) que cumplirían las poblaciones de microalgas y explicarían, en parte, su presencia en los ensamblados o asociaciones en cada momento y lugar. Algunas de estas reglas, obtenidas de la repetida observación empírica, han quedado demostradas en este trabajo y otras nuevas han surgido. Así, de entre las primeras, ha quedado probado que todas las algas en un momento y lugar tenderán a alcanzar su máximo potencial (regla 1 en Reynolds et al., 2000); en principio serán dominantes las especies de mayor tasa de crecimiento (regla 2); el ambiente selecciona ciertos atributos o caracteres de las algas (regla 4 en Reynolds et al., 2000), en este caso los factores de control o selección son del ambiente tanto abiótico como biótico; el resultado de un proceso de ensamblado de comunidades está sujeto a la depredación y otras interacciones (regla 8). Pero si nos fijamos en la última regla 10 , en la que se asegura que el ensamblado puede sufrir variaciones ambientales o re-inicializaciones, veremos la importancia que tienen algunos de los resultados aquí obtenidos y que deberíamos añadir como nuevas reglas de ensamblado o matización de las existentes. Varias reglas de ensamblado hacen referencia al pasado reciente como determinante de las especies relevantes del momento actual (Reynolds et al., 2000): la presencia mayoritaria de un alga en el momento actual puede indicar que fue la más abundante en ese medio en un pasado reciente (regla 3 ) y otra similar: las 
poblaciones de atributos mejor adaptados serán las que generen una mayor población y por tanto un gran inóculo para cuando las condiciones sean favorables (regla 5) y también que de las especies presentes la dominante es, probablemente, una especie con las adaptaciones más ventajosas (regla 9). Sin embargo, deberían ser todas ellas puntualizadas ya que, como hemos observado, la más abundante en la actualidad que venga de un inóculo pasado deberá ser aquella con el inóculo más viable tras la senescencia, y cuyas propiedades les favorezcan no sólo donde y cuando están presentes sino también, y no menos importante, con quien estén ensambladas. El propio ensamblado determina la eficacia de las poblaciones presentes, o como se expresa en la teoría de sistemas, el sistema afecta a las partes (Frontier \& Pichod-Viale, 1991).

Por otra parte, se ha podido comprobar como a partir de un mismo conjunto de especies las diferentes estructuras o ensamblados que se generan en distintas condiciones presentan diferente funcionamiento. Es decir, se ha constatado la influencia de la estructura del ensamblado sobre la función, entendida esta última como la producción fotosintética y la respiración. Así se observa que, en aquellas condiciones que favorecen la proliferación de las algas de crecimiento rápido (Scenedesmus y Monoraphidium) y alta tasa respiratoria (Olrik, 1994), las comunidades son de mayor actividad fotosintética y respiración. En cuanto al balance entre producción y respiración, los cultivos (tratándose de cultivos discontinuos, sin renovación de medio) de los diferentes tratamientos se comportaron autotróficamente $(\mathrm{PPB} / \mathrm{R}>1)$ hasta prácticamente los 30 días de incubación y solo a partir de ese momento (incluso después de un período más largo de cultivo) la mayoría de ellos lo hicieron heterotróficamente $(\mathrm{PPB} / \mathrm{R}<1)$. Esta observación está en concordancia con los datos sobre balance producción-respiración de la comunidad planctónica presentados por Carignan y colaboradores (2000) para lagos oligotróficos. Estos autores, utilizando como metodología la medida de la producción y consumo de oxígeno para determinar fotosíntesis y respiración, demostraron que el cociente fotosíntesis-respiración ex- cedía 1 en prácticamente todos los lagos estudiados. Sus resultados entran en contradicción con los obtenidos previamente por del Giorgio \& Peters (1994) quienes, a partir de la utilización del método del $\mathrm{C}^{14}$, determinaron que los cocientes PPB/R eran menores que 1 en los lagos que ellos estudiaron y concluyeron que en dichos lagos dominaba la heterotrofia. Nuestros datos sobre fotosíntesis y respiración (aunque se trate de un estudio in vitro), obtenidos con una metodología semejante a la utilizada por Carignan y colaboradores (producción y consumo de oxígeno) reforzarían las conclusiones citadas por Carignan et al., (2000) sobre la autotrofia neta de las comunidades planctónicas (epilimnéticas y metalimnéticas).

\section{CONCLUSIONES}

El ensamblado de algas que se forme a partir de un conjunto de especies dependerá de una serie de reglas de ensamblado derivadas tanto de los caracteres fisiológicos y morfológicos de las especies como del complejo sistema de interacciones que se genere. De modo que la previsión sobre cuál será el ensamblado más probable, basada en la estrategia de crecimiento de las algas, se verá alterada por el efecto del depredador y por la viabilidad del inóculo que a su vez condicionará nuevas combinaciones de competidores. En oligotrofia, la diversidad queda mantenida por la falta de founder controler (Weiher \& Keddy, 1999) y las diferentes estrategias de captación y retención de los nutrientes (Levins, 1979; Tilman, 1982). Por el contrario, en eutrofia, la presencia de buenos competidores lleva a la pérdida de diversidad. La presencia de herbívoros reduce la biomasa de los productores primarios pero además aumenta la diversidad relajando la competencia (Harris, 1986) y aumenta la incertidumbre sobre el final alcanzado ya que se incluye en las trayectorias de los ensamblados la variabilidad de la dinámica de los herbívoros, al tiempo que además, se ha aumentado la diversidad de la dieta (Pimm, 1982). Por tanto, podemos decir que la diversidad alcanzada a partir de un conjunto conocido de especies no sólo debe eva- 
luarse en cada estructura resultante de las interacciones o mecanismos subyacentes sino como el conjunto de todos los estados finales posibles (Fukami, 2004). Y por último debemos remarcar que los cambios en la estructura de las comunidades afectan en última instancia a aspectos funcionales de los ecosistemas como la producción primaria y la respiración, como ya se ha demostrado en algas marinas (Eriksson et al., 2006).

\section{AGRADECIMIENTOS}

Los autores agradecen a Miguel Álvarez el préstamo del equipo del electrodo de Clark. También se agradece al Ministerio de Educación y Ciencia la financiación del proyecto CGL200602891 y la beca AP-2004-5315 de José Larrosa.

\section{BIBLIOGRAFÍA}

CARIGNAN, R., D. PLANAS \& C. VIS. 2000. Planktonic production and respiration in oligotrophic Shield lakes. Limnol. Oceanogr., 45: 189-199.

CARPENTER, S. R. \& J. F. KITCHELL (eds.), 1993. The trophic cascade in lakes. Cambridge. Cambridge University Press. 386 pp.

CHOW-FRASER, P. 1998. A conceptual ecological model to aid restoration of Cootes Paradise Marsh, a degraded coastal wetland of Lake Ontario, Canada. Wetlands Ecol. Manage., 6. 43-57

COTTINGHAM, K. L. 1999. Nutrients and zooplankton as multiple stressors of phytoplankton communities: evidence from size structure. Limnol. Oceanogr., 44: 810-827.

COTTINGHAM, K. L., S. GLAHOLT \& A. C. BROWN. 2004. Zooplankton community structure affects how phytoplankton respond to nutrient pulses. Ecology, 85: 158-171.

DEL GIORGIO, P. A. \& R. H. PETERS. 1994. Patterns in planktonic P:R ratios: Influence of lake trophy and dissolved organic carbon. Limnol. Oceanogr., 39: 772-787.

DRAKE, J. A. 1991. Community-assembly mechanics and the structure of an experimental species ensemble. Am. Nat., 137: 1-26.

DRAKE, J. A., C. ZIMMERMAN, T. PURUCKER \& C. ROJO. 1999. On the nature of the assembly trajectory. In: Ecological Assembly Rules. E.
Weiher \& P. Keddy (eds.): 233-251. Cambridge University Press.

DUBINSKY Z., P. G. FALKOWSKI, A. F. POST \& U. M. VANHES, 1987. A system for measuring phytoplankton photosynthesis in a defined lightfield with an oxygen-electrode. J. Plank. Res., 9: 607-612.

ERIKSSON, B. K., A. RUBACH, \& H. HILLEBRAUND. 2006. Biotic habitat complexity controls species diversity and nutrient effects on net biomass production. Ecology, 87: 246-254.

FRONTIER, S. \& D. PICHOD-VIALE. 1991 Ecosystémes: structure, fonctionnement, evolution. Collection d'ecologie (21) Masson (Paris). 447 pp.

FUKAMI, T. 2004. Community assembly along a species pool gradient: implications for multiplescale patterns of species diversity. Popul. Ecol., 46: 137-147.

GLIWICZ, Z. M. 1990. Food thresholds and body size in cladocerans. Nature, 343: 638-640.

HARRIS, G. P. 1986. Phytoplankton ecology. London. Chapman \& Hall. 384 pp.

LAMPERT, W. \& U. SOMMER. 1997. Limnoecology: the Ecology of Lakes and Streams. New York. Oxford University Press. 382 pp.

LEVINS, R. 1979. Coexistence in a variable environment. Am. Nat., 114: 765-783.

MARGALEF, R. 1958. Temporal succession and spatial heterogeneity in phytoplankton. In: Perspectives in Marine Biology. A. A. Buzzati-Traverso (ed.): 323-349. Berkeley. University California Press.

NASELLI-FLORES, L., J. PADISÁK, M. DOKULIL \& I. CHORUS. 2003. Equilibrium/steadystate concept in phytoplankton ecology. Hydrobiologia, 502: 395-403.

PIMM, S. L. 1982. Food webs. London. Chapman and Hall. 219 pp.

ORTEGA-MAYAGOITIA, E., C. ROJO \& M. A. RODRIGO. 2002. Factors masking the trophic cascade in shallow eutrophic wetlands- evidence from a microcosm study. Arch. Hydrobiol., 155: 43-63.

ORTEGA-MAYAGOITIA, E., C. ROJO \& M. A. RODRIGO. 2003. Controlling factors of phytoplankton taxonomic structure in wetlands: an experimental approach. Hydrobiologia, 502: 177-186.

PADISÁK, J. 1992. Seasonal succession of phytoplankton in a large shallow lake (Balaton, Hungary)-a dynamic approach to biological memory, its possible role and mechanisms. J. Ecol,. 80: $217-230$. 
REYNOLDS, C. S. 1984. The ecology of freshwater phytoplankton. Cambridge. Cambridge University Press. 384 pp.

REYNOLDS, C. S., J. PADISÁK \& U. SOMMER. 1993. Intermediate disturbance in the ecology of phytoplankton and the maintenance of species diversity: a synthesis. Hydrobiologia, 249: 183-188.

REYNOLDS, C. S. 1997. Vegetation processes in the pelagic: a model for ecosystem theory. Excellence in Ecology. Ecology Institute. Oldendorf. 371 pp.

REYNOLDS, C.S. 1998. What factors influence composition of phytoplankton in lakes of different trophic status? Hydrobiologia, 369/370: 11-26.

REYNOLDS, C. S., M. DOKULIL \& J. PADISÁK. 2000. Understanding the assembly of phytoplank ton in relation to the trophic spectrum: where are we now? Hidrobiología, 424: 147-152.

ROJO, C., E. ORTEGA-MAYAGOITIA, M. A. RODRIGO \& M. ÁLVAREZ-COBELAS. 2000. Phytoplankton structure and dynamics in a semiarid wetland, the National Park Las Tablas de Daimiel
(Spain). Archiv. Hydrobiol., 148: 397-419.

ROJO, C. \& M. ÁLVAREZ-COBELAS. 2000. A plea for more ecology in phytoplankton ecology. Hydrobiologia, 424:141-146.

ROTT, E. 1981. Some results from phytoplankton counting intercalibrations. Schweiz. Z. Hydrol., 43:34-62.

SOMMER, U., M. Z. GLIWICZ, W. LAMPERT \& A. DUNCAN. 1986. The PEG-model of seasonal succession of planktonic events in freshwaters. Archiv. Hydrobiol., 106: 433-471.

TILMAN, D. 1982. Resource competition and community structure. Princeton. Princeton University Press. 296 pp.

VOLLENWEIDER, R. A. 1969. A manual on methods for measuring primary production in aquatic environments. IBP Handbook No 12. Oxford. Blackwell. 228 pp.

WEIHER, E. \& P. KEDDY , 1999. Ecological assembly rules. Cambridge. Cambridge University Press. 418 pp. 